\title{
Editorial
}

\section{Soft Matter Rheology: Theory and Experiments}

\section{Guest Editors, Kyu Hyun ${ }^{1}$ and Won Bo Lee ${ }^{2}$}

The term "soft matter" represents the material system whose important energy scale is comparable to thermal energy at room temperature, and thus its physical and mechanical properties are easily altered by the thermal fluctuations. The subject of soft matter includes polymers, biomaterials, surfactants, liquid crystals, colloids, glasses and granular materials, and interests on the subject continue to grow rapidly owing to its unique material properties such as tenability and responsiveness. For the last few decades, a strong research collaboration on the subject of "soft matter" has initiated among engineers, biologists, physicists and chemists, and the community of "soft matter" has been dramatically grown both in size and quality.

The facts that these "soft matter" are neither simple liquids nor crystalline solids and also have unique internal structures with micro- to nano-meter sizes prevent "soft matter" from being readily categorized into the classical scheme of materials classification. Under the processing condition, "soft matter" experiences various deformations (shear, and extensional flow, and so on), which change the fluid morphologies, e.g. its micro- or nanostructure, and therefore, the mechanical properties. This is one major reason why the study on coupling between internal structures of soft matter and flow fields catches more attention. In this regard, the subject rheology is prerequisite for understanding behaviors of soft matters and for their industrial applications.

The present (mini) special issue includes seven articles written by relatively young researchers working on the experimental or theoretical studies of soft matter rheology. They are contributed from seven different countries (Belgium, Canada, China, Greece, Korea, Taiwan, and US). We hope that this issue will help broaden Korea-Aust. Rheol. J. readers' perspective on "soft matter".

\section{Editor-in-Chief, Myung-Suk Chun ${ }^{3}$}

Work in the area of soft matters is interdisciplinary in nature, both in terms of the applications and systems under the theoretical and experimental investigations used to approach them. Rheology has opened new paths in soft matter and soft matter has driven new developments in rheology. It is our sincere hope that the readers greatly benefit from this themed issue (4 review articles and 3 original papers) for the study and manipulation of soft matters.

I would like to thank Guest Editors and all authors for their contributions, which will have a significant impact on the field. Likewise, I greatly appreciate all the referees' efforts for the quality of the publications. Finally, I have to show gratitude the Associate Editor, Hyun Wook Jung and Staff of the Journal for their continuous supports.

${ }^{1}$ School of Chemical and Biomolecular Engineering, Pusan National University, Busan 609-735

${ }^{2}$ Department of Chemical and Biomolecular Engineering, Sogang University, Seoul 121-742

${ }^{3}$ National Agenda Research Division, Korea Institute of Science and Technology (KIST), Seoul 136-791 